\title{
TWO POINT BOUNDARY PROBLEMS FOR SECOND ORDER MATRIX DIFFERENTIAL SYSTEMS
}

BY

\author{
GARRET J. ETGEN
}

\begin{abstract}
This paper is concerned with second order matrix differential systems involving a parameter together with boundary conditions specified at two points. The object of the paper is to establish sufficient conditions for the existence of eigenvalues for the system. Although such problems have been considered using the results of and techniques from the calculus of variations, the methods and results here are entirely in the context of ordinary differential equations. Use is made of the matrix generalization of the polar coordinate transformation introduced by J. H. Barrett and the unitary transformation suggested by F. V. Atkinson and V. A. Jakubovič. The sufficient conditions for the existence of eigenvalues obtained here represent certain extensions of W. M. Whyburn's work concerning linear and nonlinear boundary problems for second order differential systems.
\end{abstract}

1. Introduction. The two point boundary problem

$$
\begin{aligned}
{\left[p(x, \lambda) y^{\prime}\right]^{\prime}+q(x, \lambda) y } & =0, \\
\alpha(\lambda) y(a, \lambda)-\beta(\lambda) y^{\prime}(a, \lambda) & =0, \\
\gamma(\lambda) y(b, \lambda)-\delta(\lambda) y^{\prime}(b, \lambda) & =0,
\end{aligned}
$$

defined on $a \leqq x \leqq b, \Lambda_{1}<\lambda<\Lambda_{2}$, where each of $p(x, \lambda)$ and $q(x, \lambda)$ is a continuous, real-valued function with $p>0$, is known as a Sturmian system and it has been studied in considerable detail. In addition to the classical works of Sturm and Liouville, we refer to the treatments contained in F. V. Atkinson [1], E. A. Coddington and N. Levinson [3], and E. L. Ince [8]. Linear and nonlinear versions of the Sturmian system together with more general boundary conditions have been considered by W. M. Whyburn [7], [14] and [15].

The object of this paper is to consider a corresponding second order matrix differential system

$$
Y^{\prime}=K(x, \lambda) Z, \quad Z^{\prime}=-G(x, \lambda) Y
$$

defined on $X: a \leqq x \leqq b, L: \lambda_{0}-\delta<\lambda<\lambda_{0}+\delta$ ( $\delta$ may be infinite) where each of $K$ and $G$ is an $n \times n$ symmetric matrix of continuous, real-valued functions on

Received by the editors August 29, 1969.

AMS Subject Classifications. Primary 3430, 3436, 3442.

Key Words and Phrases. Matrix differential equations, second order matrix differential systems, two point boundary problems, existence of eigenvalues, oscillatory behavior.

Copyright (C) 1970, American Mathematical Society 
$X L$ with $K$ being positive definite. We shall be concerned with the solution pairs $\{Y(x, \lambda), Z(x, \lambda)\}$ of $(1.1)$ which satisfy the two point boundary conditions

$$
\begin{aligned}
A^{*}(\lambda) Y(a, \lambda)-B^{*}(\lambda) Z(a, \lambda) & \equiv 0, \\
\operatorname{det}\left[\Gamma^{*}(\lambda) Y(b, \lambda)-\Delta^{*}(\lambda) Z(b, \lambda)\right] & =0
\end{aligned}
$$

(* denotes transpose) where $A(\lambda), B(\lambda), \Gamma(\lambda)$ and $\Delta(\lambda)$ are $n \times n$ matrices of continuous, real-valued functions on $L$. A primary reason for considering the boundary conditions in this form is presented in $\$ 2$ where it is shown that the two point boundary problem (1.1), (1.2) has a nontrivial solution if and only if the corresponding vector-matrix two point boundary problem

$$
\begin{gathered}
y^{\prime}=K(x, \lambda) z, \quad z^{\prime}=-G(x, \lambda) y, \\
A^{*}(\lambda) y(a, \lambda)-B^{*}(\lambda) z(a, \lambda)=0, \\
\Gamma^{*}(\lambda) y(b, \lambda)-\Delta^{*}(\lambda) z(b, \lambda)=0
\end{gathered}
$$

has a nontrivial solution.

Two point boundary problems of the form (1.1), (1.2) have been studied extensively using techniques and results from the calculus of variations. We refer in particular, to M. Morse [10] and W. T. Reid [11], [12]. Our approach here is independent of the calculus of variations and our results are along the lines indicated in [7] and [15].

The basic techniques are derived from the methods developed by Atkinson [1, Chapter 10] and V. A. Jakubovič [9]. In addition, we employ the matrix generalization of the polar coordinate, or Prüfer, transformation as introduced by J. H. Barrett [2] and subsequently refined by Reid [13]. The manner in which this transformation is applied has been suggested by Whyburn's work.

The notations of matrix algebra and calculus are used throughout. Generally, capital letters are used to denote matrices while lower case letters denote scalar quantities. Most of the matrices to be considered are $n \times n$ and, consequently, if the term "matrix" is used it is assumed to be $n \times n$. The symbol $I$ is used to signify the identity matrix and 0 is used for the zero matrix, regardless of dimension. If $M$ is a matrix, then $M^{*}$ shall denote the transpose of $M, \operatorname{tr} M$ the trace of $M$, det $M$ the determinant of $M$, and in the event det $M \neq 0, M^{-1}$ shall denote the inverse of $M$. The notation $M \geqq N(M>N)$ is used to signify that each of $M$ and $N$ is symmetric with $M-N$ nonnegative (positive) definite. Finally, if $Y(x)$ is a matrix each of whose entries is a function, then $Y(x)$ will be termed continuous, differentiable, etc., when each of its entries has the indicated property.

2. Definitions and preliminary remarks. We begin by listing the basic properties which we assume the coefficients in the system possess:

H.1. Each of $K(x, \lambda)$ and $G(x, \lambda)$ is continuous and symmetric on $X L$.

H.2. $K(x, \lambda)$ is positive definite on $X L$.

H.3. $A^{*}(\lambda) B(\lambda) \equiv B^{*}(\lambda) A(\lambda)$ on $L$. 
H.4. $\Gamma^{*}(\lambda) \Delta(\lambda) \equiv \Delta^{*}(\lambda) \Gamma(\lambda)$ on $L$.

We shall also assume that $A^{*} A+B^{*} B>0$ and $\Gamma^{*} \Gamma+\Delta^{*} \Delta>0$ on $L$. However, with these assumptions, we note that we can, without loss of generality, assume:

H.5. $A^{*}(\lambda) A(\lambda)+B^{*}(\lambda) B(\lambda) \equiv I$ on $L$.

H.6. $\Gamma^{*}(\lambda) \Gamma(\lambda)+\Delta^{*}(\lambda) \Delta(\lambda) \equiv I$ on $L$.

Definition 2.1. A solution pair $\{Y(x, \lambda), Z(x, \lambda)\}$ on (1.1) is conjoined provided

$$
Y^{*}(x, \lambda) Z(x, \lambda) \equiv Z^{*}(x, \lambda) Y(x, \lambda)
$$

on $X$ for each $\lambda$ on $L$. (See Morse [10, Chapter III].)

DEFINITION 2.2. A solution pair $\{Y(x, \lambda), Z(x, \lambda)\}$ of (1.1) is nontrvial provided det $Y(x, \lambda)$ has at most a finite number of zeros on $X$ for each fixed $\lambda$ on $L$.

A characterization of the nontrivial, conjoined solution pairs of (1.1) is given by the following:

Theorem A (Atkinson [1], Chapter 10). Fix any $\lambda$ on L. A necessary and sufficient condition that a solution pair $\{Y(x, \lambda), Z(x, \lambda)\}$ of $(1.1)$ be conjoined and nontrivial is that there exist numbers $c$ and $d, a \leqq c, d \leqq b$, such that

(i) $Y^{*}(c, \lambda) Z(c, \lambda)=Z^{*}(c, \lambda) Y(c, \lambda)$,

(ii) $Y^{*}(d, \lambda) Y(d, \lambda)+Z^{*}(d, \lambda) Z(d, \lambda)>0$.

(See also Morse [10, Chapter III, §3].)

Definition 2.3. The values of $\lambda$ for which there exists a nontrivial, conjoined solution pair of (1.1) satisfying (1.2) are called the eigenvalues of the system.

We show here that the problem (1.1), (1.2) has a nontrivial conjoined solution if and only if the vector-matrix two point boundary problem $(1.1)^{\prime},(1.2)^{\prime}$ has a nontrivial solution. We demonstrate this by supposing that for $\lambda=\lambda_{1},\left\{Y\left(x, \lambda_{1}\right)\right.$, $\left.Z\left(x, \lambda_{1}\right)\right\}$ is a nontrivial solution of $(1.1),(1.2)$. Then, since

$$
\operatorname{det}\left[\Gamma^{*}\left(\lambda_{1}\right) Y\left(b, \lambda_{1}\right)-\Delta^{*}\left(\lambda_{1}\right) Z\left(b, \lambda_{1}\right)\right]=0,
$$

there exists a nonzero, constant vector $\gamma$ such that

$$
\left[\Gamma^{*}\left(\lambda_{1}\right) Y\left(b, \lambda_{1}\right)-\Delta^{*}\left(\lambda_{1}\right) Z\left(b, \lambda_{1}\right)\right] \gamma=0 .
$$

It is now readily verified that $\left\{Y\left(x, \lambda_{1}\right) \gamma, Z\left(x, \lambda_{1}\right) \gamma\right\}$ is a nontrivial solution of $(1.1)^{\prime},(1.2)^{\prime}$. On the other hand, let $\lambda=\lambda_{2}$ be a value of $\lambda$ such that $\left\{y\left(x, \lambda_{2}\right)\right.$, $\left.z\left(x, \lambda_{2}\right)\right\}$ is a nontrivial solution of $(1.1)^{\prime},(1.2)^{\prime}$. The solution pair $\left\{Y\left(x, \lambda_{2}\right)\right.$, $\left.Z\left(x, \lambda_{2}\right)\right\}$ of (1.1) having the initial value $Y\left(a, \lambda_{2}\right)=B\left(\lambda_{2}\right), Z\left(a, \lambda_{2}\right)=A\left(\lambda_{2}\right)$ satisfies (1.2a). Let $\gamma=y\left(a, \lambda_{2}\right), \delta=z\left(a, \lambda_{2}\right)$ and put $\beta=B^{*}\left(\lambda_{2}\right) \gamma+A^{*}\left(\lambda_{2}\right) \delta$. Then, $\beta \neq 0$, $Y\left(x, \lambda_{2}\right) \beta \equiv y\left(x, \lambda_{2}\right), Z\left(x, \lambda_{2}\right) \beta \equiv z\left(x, \lambda_{2}\right)$. Now, the fact that $\left\{y\left(x, \lambda_{2}\right), z\left(x, \lambda_{2}\right)\right\}$ satisfies (1.2b)' implies $\Gamma^{*}\left(\lambda_{2}\right) Y\left(b, \lambda_{2}\right) \beta-\Delta^{*}\left(\lambda_{2}\right) Z\left(b, \lambda_{2}\right) \beta=0$ from which it follows that the pair $\left\{Y\left(x, \lambda_{2}\right), Z\left(x, \lambda_{2}\right)\right\}$ satisfies $(1.2 b)$.

As a result of the conditions which $A$ and $B$ were assumed to possess, we conclude, from Theorem $\mathrm{A}$, that the solution pair $\{Y(x, \lambda), Z(x, \lambda)\}$ of $(1.1)$ satisfying

$$
Y(a, \lambda) \equiv B(\lambda), \quad Z(a, \lambda) \equiv A(\lambda) \quad \text { on } L,
$$


is nontrivial and conjoined. Clearly, this pair satisfies (1.2a). We shall now show that this pair is the only solution which we need consider. First, note that (1.1), (1.2a) does not have a unique solution; for if $\{U, V\}$ is a solution, then $\{U Q, V Q\}$, $Q$ a constant matrix, is also a solution. We prove, however,

LEMMA 2.1. Let $\{Y(x, \lambda), Z(x, \lambda)\}$ be the solution of $(1.1),(2.1)$ and let $\{U(x, \lambda)$, $V(x, \lambda)\}$ be any other solution of (1.1) satisfying (1.2a). Then, for each $\lambda$ on $L$ there exists a constant matrix $N$ (depending on the pair $\{U, V\})$ such that $U(x, \lambda) \equiv Y(x, \lambda) N$, $V(x, \lambda) \equiv Z(x, \lambda) N$ on $X$.

Proof. As observed above, the pair $\{Y(x, \lambda), Z(x, \lambda)\}$ is nontrivial and conjoined. Fix any $\lambda$ on $L$, let $c$ be a point at which det $Y(c) \neq 0$, and consider the matrix $F=Y^{-1} U$ defined on some interval $d \leqq x \leqq e$ containing $c$. Calculating the derivative, we have

$$
\begin{aligned}
F^{\prime} & =Y^{-1} U^{\prime}-Y^{-1} Y^{\prime} Y^{-1} U \\
& =Y^{-1} K\left(V-Z Y^{-1} U\right) .
\end{aligned}
$$

Since $Y^{*} Z \equiv Z^{*} Y$ and $Y^{*} V-Z^{*} U \equiv 0$, it follows that $V-Z Y^{-1} U=0$. Thus $F^{\prime}=0$ and $F=N$, a constant matrix, on $d \leqq x \leqq e$. It now follows that $U \equiv Y N$ and $V \equiv Z N$ on $X$.

One of the principal methods to be employed in this paper is based on the result contained in [1, Chapter $10, \S \S 2-5]$ and in [6]. These results are summarized by the following theorem.

THEOREM B. Let $\{Y(x, \lambda), Z(x, \lambda)\}$ be the solution pair of (1.1), (1.2). The matrix $\theta(Y, Z)$ defined by

$$
\theta(Y, Z)=(Z+i Y)(Z-i Y)^{-1}, \quad i^{2}=-1,
$$

exists on $X L$ and for each $\lambda$ on $L$, has the following properties on $X$ :

(i) $\theta$ is a unitary matrix.

(ii) $\theta$ satisfies the differential equation

$$
\theta^{\prime}=2 i \theta \Omega(x, \lambda)
$$

where

$$
\begin{aligned}
\Omega(x, \lambda) & =\left(Z^{*}+i Y^{*}\right)^{-1}\left[Z^{*} Y^{\prime}-Y^{*} Z^{\prime}\right]\left(Z-i Y^{*}\right)^{-1} \\
& =\left(Z^{*}+i Y^{*}\right)^{-1}\left[Z^{*} K Z+Y^{*} G Y\right](Z-i Y)^{-1}
\end{aligned}
$$

(iii) Let $\phi_{j}(x, \lambda), j=1,2, \ldots, n$, denote the characteristic roots of $\theta$. Then $\left|\phi_{j}(x, \lambda)\right|=1, j=1,2, \ldots, n$, on $X$ and for any fixed $x, \phi_{j}(x, \lambda)=+1$ for at least one $j$ if and only if det $Y(x, \lambda)=0$.

(iv) The functions $\phi_{j}(x, \lambda)$ move monotonically and positively on the unit circle when they are at the point +1 .

(v) For each fixed $x$ on $X$, the multiplicity of a zero of $\operatorname{det} Y(x, \lambda)$, i.e. the 
dimension of the null space of $Y(x, \lambda)$, is equal to the number of characteristic roots $\phi_{j}(x, \lambda)$ of $\theta$ having the value +1 .

(vi) Let $w_{j}(x, \lambda)=\arg \phi_{j}(x, \lambda), j=1,2, \ldots, n$, where it is assumed that $0 \leqq w_{j}\left(a, \lambda_{0}\right)$ $<2 \pi$ for each $j$ and that the functions $w_{j}(x, \lambda)$ are continued as continuous functions on XL. Then

$$
\operatorname{det} \theta=\exp \left\{i \sum_{j=1}^{n} w_{j}(x, \lambda)\right\}
$$

and

$$
2 \int_{a}^{x} \operatorname{tr} \Omega(t, \lambda) d t=\sum_{j=1}^{n}\left[w_{j}(x, \lambda)-w_{j}(a, \lambda)\right]
$$

3. The polar coordinate transformation. The solution pair $\{Y(x, \lambda), Z(x, \lambda)\}$ of (1.1), (2.1) clearly satisfies (1.2a) and consequently it is now necessary to establish the existence of values of $\lambda$ for which this pair also satisfies (1.2b) To accomplish this it is convenient to employ a polar coordinate transformation.

A matrix generalization of the trigonometric functions, introduced by $\mathbf{J}$. $\mathbf{H}$. Barrett [2], is obtained by considering the solution pairs $\{S(x), C(x)\}$ of the matrix differential system.

$$
\begin{aligned}
Y^{\prime}(x) & =Q(x) Z(x), & Z^{\prime}(x) & =-Q(x) Y(x) \text { on } X, \\
Y(a) & =M, & Z(a) & =N,
\end{aligned}
$$

where $Q(x)$ is an $n \times n$ continuous symmetric matrix and $M$ and $N$ are $n \times n$ constant matrices satisfying $M^{*} N=N^{*} M, M^{*} M+N^{*} N=I$. The author [4], [5], has established that the solution pairs of systems of the form (3.1), (3.2) behave in a manner quite similar to the trigonometric functions.

The application of the polar coordinate transformation to the solutions of matrix systems of the form (1.1) was first presented by Barrett [2] and was subsequently refined by Reid [13]. The next theorem is an immediate extension of their work.

TheOrem C. Let $\{Y(x, \lambda), Z(x, \lambda)\}$ be the solution pair of (1.1), (2.1). There exists a continuous, symmetric matrix $Q(x, \lambda)$ and a nonsingular, continuously differentiable (in $x)$ matrix $R(x, \lambda)$ on $X L$ such that

$$
Y(x, \lambda)=S^{*}(x, \lambda) R(x, \lambda), \quad Z(x, \lambda)=C^{*}(x, \lambda) R(x, \lambda)
$$

on $X$ for each $\lambda$ on $L$, where $\{S(x, \lambda), C(x, \lambda)\}$ is the solution of

$$
\begin{aligned}
S^{\prime} & =Q(x, \lambda) C, & C^{\prime} & =-Q(x, \lambda) S, \\
S(a, \lambda) & =B^{*}(\lambda), & C(a, \lambda) & =A^{*}(\lambda) .
\end{aligned}
$$

Moreover, $R(x, \lambda)$ is the solution of

$$
R^{\prime}=\left[S K C^{*}-C G S^{*}\right] R, \quad R(a, \lambda)=I
$$

and

$$
Q(x, \lambda)=C K C^{*}+S G S^{*} \text {. }
$$


Now, using Theorem C, the boundary form (1.2b) may be written in terms of the matrices $S$ and $C$ yielding

$$
\Gamma^{*} Y-\Delta^{*} Z=\Gamma^{*} S^{*} R-\Delta^{*} C^{*} R=\left[\Gamma^{*} S^{*}-\Delta^{*} C^{*}\right] R .
$$

Since $R$ is nonsingular on $X L$, the zeros of det $\left[\Gamma^{*} Y-\Delta^{*} Z\right]$ coincide with those of $\operatorname{det}\left[\Gamma^{*} S^{*}-\Delta^{*} C^{*}\right]$. Define the pair of matrices $\{U, V\}$ by the equations

$$
\begin{aligned}
& U(x, \lambda)=S(x, \lambda) \Gamma(\lambda)-C(x, \lambda) \Delta(\lambda), \\
& V(x, \lambda)=C(x, \lambda) \Gamma(\lambda)+S(x, \lambda) \Delta(\lambda) .
\end{aligned}
$$

It is readily verified that the pair $\{U, V\}$ is a trigonometric pair in the sense that this pair is the solution of (3.1), (3.2) where $Q(x, \lambda)$ is given by (3.5) and

$$
\begin{gathered}
M(\lambda)=B^{*}(\lambda) \Gamma(\lambda)-A^{*}(\lambda) \Delta(\lambda), \\
N(\lambda)=A^{*}(\lambda) \Gamma(\lambda)+B^{*}(\lambda) \Delta(\lambda),
\end{gathered}
$$

where for each fixed $\lambda, M$ and $N$ have the required properties, namely $M^{*} N=N^{*} M$, $M^{*} M+N^{*} N=I$.

Since an initial value problem defining a pair of trigonometric matrices is, essentially, an initial value problem of the form (1.1), (2.1), it is readily verified that each of the matrices

$$
E(X, \lambda)=(C-i S)^{-1}(C+i S)
$$

and

$$
F(x, \lambda)=(V-i U)^{-1}(V+i U),
$$

where $\{S, C\}$ is given by (3.3), (3.4) and $\{U, V\}$ by (3.6), exists on $X L$ and has properties (i)-(iii), (v) and (vi) of Theorem B. Moreover, using Theorem C, it is easily established that $E(x, \lambda) \equiv \theta(x, \lambda)$ on $X L$ and hence $E$ also possesses property (iv) of Theorem B. Let $f_{j}(x, \lambda), j=1,2, \ldots, n$, denote the characteristic roots of $F(x, \lambda)$ and let $\beta_{j}(x, \lambda)=\arg f_{j}(x, \lambda)$ for each $j$, with the assumptions that $0 \leqq \beta_{j}\left(a, \lambda_{0}\right)<2 \pi$ and $\beta_{j}(x, \lambda)$ is continued as a continuous function on $X L$. Quoting identities (2.5) and (2.6), we have

$$
\begin{aligned}
& \operatorname{det} E(x, \lambda)=\exp \left\{i \sum_{j=1}^{n} w_{j}(x, \lambda)\right\}, \\
& \operatorname{det} F(x, \lambda)=\exp \left\{i \sum_{j=1}^{n} \beta_{j}(x, \lambda)\right\},
\end{aligned}
$$

and

$$
\begin{aligned}
2 \int_{a}^{x} \operatorname{tr} Q(t, \lambda) d t & =\sum_{j=1}^{n}\left[w_{j}(x, \lambda)-w_{j}(a, \lambda)\right]=\sum_{j=1}^{n}\left[\beta_{j}(x, \lambda)-\beta_{j}(a, \lambda)\right] \\
& =\int_{a}^{x} \operatorname{tr} \Omega(t, \lambda) d t
\end{aligned}
$$

on $X L$. 
4. Existence of eigenvalues. In this section we will be concerned with establishing conditions which will insure the existence of eigenvalues for the system (1.1), (1.2), as indicated in $\S 3$. The zeros of det $\left[\Gamma^{*}(\lambda) Y(x, \lambda)-\Delta^{*}(\lambda) Z(x, \lambda)\right]$ coincide with those of det $U(x, \lambda)$ defined by (3.6). In particular then, we shall be concerned with the behavior of the functions $\beta_{j}(x, \lambda)$, the arguments of the characteristic roots of $F(x, \lambda)$. More specifically, the eigenvalues for the system are the values of $\lambda$ for which $\beta_{j}(b, \lambda) \equiv 0(\bmod 2 \pi)$ for at least one $j$.

Atkinson [1, Chapter 10] has established the following inequalities which hold on $X L$ :

$$
\begin{aligned}
& 2 \operatorname{tr} \Omega(x, \lambda) \leqq \operatorname{tr}\{K(x, \lambda)+G(x, \lambda)+[K(x, \lambda)-G(x, \lambda)]\}, \\
& 2 \operatorname{tr} \Omega(x, \lambda) \geqq \operatorname{tr}\{K(x, \lambda)+G(x, \lambda)-[K(x, \lambda)-G(x, \lambda)]\},
\end{aligned}
$$

where $\Omega(x, \lambda)$ is given by $(2.4)$ and $[K-G]$ denotes the nonnegative definite square root of $(K-G)^{2}$.

Definition 4.1. For each $\lambda$ on $L$, let

$$
\begin{aligned}
& u(\lambda)=\text { g.l.b. } \operatorname{tr}\{K(x, \lambda)+G(x, \lambda)-[K(x, \lambda)-G(x, \lambda)]\}, \\
& v(\lambda)=\text { l.u.b. } \operatorname{tr}\{K(x, \lambda)+G(x, \lambda)+[K(x, \lambda)-G(x, \lambda)]\}
\end{aligned}
$$

on $X$.

For our first existence theorem, we shall impose the following two assumptions in addition to H.1-H.6 listed in $\$ 2$.

H.7. $\operatorname{det} B(\lambda) \neq 0$ on $L$.

H.8. $\operatorname{det}\left[\Gamma^{*}(\lambda) B(\lambda)-\Delta^{*}(\lambda) A(\lambda)\right] \neq 0$.

Assumptions H.7 and H.8 together with the assumption $0 \leqq w_{j}\left(a, \lambda_{0}\right), \beta_{j}\left(a, \lambda_{0}\right)$ $<2 \pi$ imply that $0<w_{j}(a, \lambda), \beta_{j}(a, \lambda)<2 \pi$ on $L$. In addition, since, for each $\lambda$, the characteristic roots $\phi_{j}(x, \lambda)$ of $\theta(Y, Z)$ pass through the point +1 in the positive direction only, $w_{j}(x, \lambda)>0$ on $X$. Thus

$$
\sum_{j=1}^{n}\left[w_{j}(x, \lambda)-w_{j}(a, \lambda)\right]=\sum_{j=1}^{n}\left[\beta_{j}(x, \lambda)-\beta_{j}(a, \lambda)\right]>-2 n \pi
$$

on $X L$. Consequently, $v(\lambda)(b-a)>-2 n \pi$ on $L$.

THEOREM 4.1. Let H.1-H.8 hold. Let $\{Y(x, \lambda), Z(x, \lambda)\}$ be the solution of (1.1), (2.1) and let $h$ be the smallest integer such that g.1.b. $v(\lambda)$ on $L$ is less than $2 h \pi /(b-a)$. If $m$ is an integer such that l.u.b. $u(\lambda)$ on $L$ is greater than $2 m n \pi /(b-a)$ and such that $m$ is greater than $h+n$ with $k$ the largest nonnegative integer having the property $m-(h+n)>k n$, then there are at least $k$ nonempty sets of eigenvalues $S_{0}, S_{1}, \ldots$, $S_{k-1}$ for the system (1.1), (1.2).

Proof. Let $Y(x, \lambda), Z(x, \lambda)$ be the solution of (1.1), (2.1) and let $\{S, C\},\{U, V\}$, $E, F$, be as described in $§ 3$. From (3.8)

$$
\sum_{j=1}^{n}\left[\beta_{j}(b, \lambda)-\beta_{j}(a, \lambda)\right]=2 \int_{a}^{b} \operatorname{tr} \Omega(t, \lambda) d t .
$$


Using the fact that $0<\beta_{j}(a, \lambda)<2 \pi, j=1,2, \ldots, n$, we have from (3.8)

$$
2 \int_{a}^{b} \operatorname{tr} \Omega(t, \lambda) d t<\sum_{j=1}^{n} \beta_{j}(b, \lambda)<2 \int_{a}^{b} \operatorname{tr} \Omega(t, \lambda) d t+2 n \pi .
$$

Let $h$ be the integer such that g.l.b. $v(\lambda)$ on $L$ is less than $2 h \pi /(b-a)$ and suppose there exists an integer $m$ with the properties indicated in the hypothesis. Then, we can conclude that there exists a $\lambda=\lambda$ on $L$ such that $v(\lambda)<2 h \pi /(b-a)$ and a $\lambda=\lambda^{*}$ on $L$ such that $u\left(\lambda^{*}\right)>2 m n \pi /(b-a)$. Clearly $\lambda \neq \lambda^{*}$ so we shall assume that $\lambda<\lambda^{*}$.

Now

$$
\sum_{j=1}^{n} \beta_{j}(b, \bar{\lambda})<2(h+n) \pi
$$

and

$$
\sum_{j=1}^{n} \beta_{j}\left(b, \lambda^{*}\right)>2 m n \pi
$$

Denoting by $k$ the largest nonnegative integer such that $m-(h+n)>k n$ and using the continuity of $\sum_{j=1}^{n} \beta_{j}(b, \lambda)$ in $\lambda$, there exist $k+1$ values of $\lambda, \lambda^{0}, \lambda^{1}, \lambda^{2}, \ldots$, $\lambda^{k}$, on the interval $\lambda<\lambda<\lambda^{*}$ such that

$$
\sum_{j=1}^{n} \beta_{j}\left(b, \lambda^{p}\right)=2(h+n+p n) \pi, \quad p=0,1, \ldots, k .
$$

Moreover, these values of $\lambda$ may be selected such that $\lambda^{0}<\lambda^{1}<\cdots<\lambda^{k}$.

Now, as $\lambda$ increases from $\lambda^{p}$ to $\lambda^{p+1}, \sum_{j=1}^{n} \beta_{j}(b, \lambda)$ increases from $2(h+n+p n) \pi$ to $2(h+n+(p+1) n) \pi$, i.e. increases by $2 n \pi$. Consequently, for at least one $j$, $\beta_{j}(b, \lambda)$ must increase by at least $2 \pi$. Therefore, there exists a value of $\lambda, \lambda^{p}<\lambda \leqq \lambda^{p+1}$ such that $\beta_{j}(b, \lambda) \equiv 0(\bmod 2 \pi)$. This value of $\lambda$ is an eigenvalue for the system (1.1), (1.2). Let $S_{p}$ be the set of all $\lambda$ 's on the interval $\lambda^{p}<\lambda \leqq \lambda^{p+1}$ such that $\beta_{j}(b, \lambda) \equiv 0$ $(\bmod 2 \pi)$ for at least one $j$. As indicated $S_{p}$ is nonempty for each $p, 0 \leqq p \leqq k-1$ and the theorem is established.

We note the following possibilities concerning the eigenvalues for the system (1.1), (1.2): first, there may exist additional sets of eigenvalues $\lambda$ outside the interval $\lambda^{0} \leqq \lambda \leqq \lambda^{k}$; second, the set $S_{p}, 0 \leqq p \leqq k-1$, may be finite, countable, uncountable, or, in fact, contain an interval.

COROLlaRY 1. Under the hypotheses of the theorem, there exist $k$ nonempty sets of eigenvalues $T_{0}, T_{1}, \ldots, T_{k-1}$ for the system (1.1), (1.2) such that if $\lambda_{p} \in T_{p}$, $0 \leqq p \leqq k-1$, then

$$
2(h+n+p n) \pi \leqq \sum_{j=1}^{n} \beta_{j}\left(b, \lambda_{p}\right)
$$

Proof. Use the continuity of $\sum_{j=1}^{n} \beta_{j}(b, \lambda)$ in $\lambda$ and the fact that $\sum_{j=1}^{n} \beta_{j}(b, \lambda)$ increases from less than $2(h+n) \pi$ to more than $2 m n \pi$, to select $\lambda^{p}$ such that for $\lambda \geqq \lambda^{p}$,

$$
\sum_{j=1}^{n} \beta_{j}(b, \lambda) \geqq 2(h+n+p n) \pi, \quad p=0,1, \ldots, k-1
$$


Let $T_{p}$ be the set of all eigenvalues on the interval $\lambda^{p}<\lambda \leqq \lambda^{p+1}, p=0,1, \ldots, k-1$. As in the proof of the theorem, each $T_{p}$ is nonempty.

COROLlaRY 2. Under the hypotheses of the theorem, if the integer $m$ may be chosen arbitrarily large, then there exist infinitely many nonempty sets of eigenvalues $S_{0}, S_{1}, \ldots$ for the system (1.1), (1.2).

COROLlaRY 3. Under the hypotheses of the theorem, let the sets of eigenvalues $T_{0}, T_{1}, \ldots, T_{k-1}$, be obtained using Corollary 1. If $k>2 \lambda_{p} \in T_{p}, p \geqq 2$, then det $Y\left(x, \lambda_{p}\right)$ has at least $p-1$ zeros on $X$, multiple zeros being counted according to their multiplicity.

Proof. As established in [6], if, for fixed $\lambda$,

$$
\sum_{j=1}^{n}\left[w_{j}(b, \lambda)-w_{j}(a, \lambda)\right] \geqq 2 r n \pi
$$

$r$ a nonnegative integer, then det $Y(x, \lambda)$ has at least $r$ zeros on $X$. Choose any $p$, $2 \leqq p \leqq k-1$, and let $\lambda_{p} \in T_{p}$. Using (3.8) and the facts that $0<\beta_{j}(a, \lambda)<2 \pi$ on $L$ and $h>-n$,

$$
\begin{aligned}
\sum_{j=1}^{n}\left[w_{j}\left(b, \lambda_{p}\right)-w_{j}\left(a, \lambda_{p}\right)\right] & =\sum_{j=1}^{n}\left[\beta_{j}\left(b, \lambda_{p}\right)-\beta_{j}\left(a, \lambda_{p}\right)\right] \\
& >2(h+n+p n) \pi-2 n \pi \\
& >2(p-1) n \pi .
\end{aligned}
$$

We now consider hypotheses for the coefficients of the system (1.1), (1.2) which will produce a monotone behavior in $\lambda$ in the solution $\{Y(x, \lambda), Z(x, \lambda)\}$ of $(1.1)$, (2.1) and in the pair $\{U(x, \lambda), V(x, \lambda)\}$ given by (3.6).

H.9. $K_{\lambda}(x, \lambda) \geqq 0$ and $G_{\lambda}(x, \lambda)>0$ (the subscript denotes differentiation with respect to $\lambda$ ) on $X L$.

H.10. $A^{*}(\lambda) B_{\lambda}(\lambda)-B^{*}(\lambda) A_{\lambda}(\lambda) \geqq 0$.

H.11. $\Gamma_{\lambda}(\lambda) \Delta^{*}(\lambda)-\Delta_{\lambda}(\lambda) \Gamma^{*}(\lambda) \geqq 0$.

H.12. $\Gamma_{\lambda}(\lambda) \Gamma^{*}(\lambda)-\Delta_{\lambda}(\lambda) \Delta^{*}(\lambda)=0$.

Hypothesis H.12 will hold, for example, if each of $\Gamma$ and $\Delta$ is independent of $\lambda$.

THEOREM 4.2. Let H.1-H.12 hold. Let $\{Y(x, \lambda), Z(x, \lambda)\}$ be the solution of (1.1), (2.1) and let $\theta(Y, Z)$ and $F(x, \lambda)$ be as defined by (2.2) and (3.7), respectively. Then, for each $x$ on $X$, each of the argument functions $w_{j}(x, \lambda)$ and $\beta_{j}(x, \lambda), j=1,2, \ldots$, $n$, of the characteristic roots of $\theta$ and $F$, is increasing in $\lambda$ on $L$.

Proof. Consider the functions $w_{j}(x, \lambda), j=1,2, \ldots, n$. Fixing $x$ and differentiating $\theta$ with respect to $\lambda$, we obtain $\theta_{\lambda}=2 i \theta H(x, \lambda)$ where

$$
H(x, \lambda)=\left(Z^{*}+i Y^{*}\right)^{-1}\left[Z^{*} Y_{\lambda}-Y^{*} Z_{\lambda}\right](Z-i Y)^{-1}
$$


Now,

$$
Y_{\lambda}^{\prime}=K_{\lambda} Z+K Z_{\lambda}, \quad Z_{\lambda}^{\prime}=-G_{\lambda} Y-G Y_{\lambda}
$$

and

$$
Y_{\lambda}(a, \lambda)=A_{\lambda}, \quad Z_{\lambda}(a, \lambda)=B_{\lambda} .
$$

Noting that

$$
\left[Z^{*} Y_{\lambda}-Y^{*} Z_{\lambda}\right]^{\prime}=Z^{*} K_{\lambda} Z+Y^{*} G_{\lambda} Y
$$

and

$$
\left[Z^{*} Y_{\lambda}-Y^{*} Z_{\lambda}\right](a, \lambda)=A^{*} B_{\lambda}-B^{*} A_{\lambda},
$$

we have, on integrating from $a$ to $x$,

$$
\left[Z^{*} Y_{\lambda}-Y^{*} Z_{\lambda}\right]=\int_{a}^{x}\left[Z^{*} K_{\lambda} Z+Y^{*} G_{\lambda} Y\right]+A^{*} B_{\lambda}-B^{*} A_{\lambda} .
$$

Thus $H(x, \lambda)>0$ on $L$ for each fixed $x$. Consequently, as shown in [1, Chapter 10], each of the functions $w_{j}(x, \lambda)$ are increasing in $\lambda$.

To verify that the functions $\beta_{j}(x, \lambda)$ are increasing on $L$, consider

$$
\begin{aligned}
F(x, \lambda) & =(V-i U)^{-1}(V+i U) \\
& =\left(V^{*}+i U^{*}\right)\left(V^{*}-i U^{*}\right)^{-1} \\
& =(\psi+i \phi)(\psi-i \phi)^{-1}
\end{aligned}
$$

where $\phi=\Gamma^{*} Y-\Delta^{*} Z, \psi=\Gamma^{*} Z+\Delta^{*} Y$. Differentiating with respect to $\lambda$, we obtain $F_{\lambda}=2 i F J(x, \lambda)$ where

$$
J(x, \lambda)=\left(\psi^{*}+i \phi^{*}\right)^{-1}\left[\psi^{*} \phi_{\lambda}-\phi^{*} \psi_{\lambda}\right](\psi-i \phi)^{-1} .
$$

From the definitions of $\phi$ and $\psi$, and the properties of $\Gamma$ and $\Delta$, we have

$$
\psi^{*} \phi_{\lambda}-\phi^{*} \psi_{\lambda}=Z^{*} Y_{\lambda}-Y^{*} Z_{\lambda}+Y^{*}\left(\Gamma_{\lambda} \Delta^{*}-\Delta_{\lambda} \Gamma^{*}\right) Y+Z^{*}\left(\Gamma_{\lambda} \Delta^{*}-\Delta_{\lambda} \Gamma^{*}\right) Z .
$$

Thus $\psi^{*} \phi_{\lambda}-\phi^{*} \psi_{\lambda}>0$ and as above, the functions $\beta_{j}(x, \lambda)$ are increasing on $L$.

With hypotheses H.9-H.12 implying monotone behavior in $\lambda$, we have the following refinement of Theorem 4.1.

THEOREM 4.3. Let H.1-H.12 hold. Let $\{Y(x, \lambda), Z(x, \lambda)\}$ be the solution of (1.1), (2.1) and let $h$ be the smallest integer such that g.l.b. $v(\lambda)$ on $L$ is less than $2 h \pi /(b-a)$. If $m$ is an integer such that l.u.b. $u(\lambda)$ on $L$ is greater than $2 m n \pi /(b-a)$ and such that $m>h+n$ with $k$ the largest nonnegative integer having the property $m-(h+n) \geqq k n$, then there exist at least $k$ nonempty sets of eigenvalues $J_{0}, J_{1}, \ldots, J_{k-1}$ for the system (1.1), (1.2). Moreover, if $\lambda_{p} \in J_{p}, 0 \leqq p \leqq k-1$, then

$$
2[h+n+p n] \pi<\sum_{j=1}^{n} \beta_{j}\left(b, \lambda_{p}\right) \leqq 2[h+n+(p+1) n] \pi .
$$

Proof. Proceeding as in the proof of Theorem 4.1, let the integers $h$ and $m$ be as described in the hypothesis. There exists $\lambda$ on $L$ such that $v(\lambda)<2 h \pi /(b-a)$ and 
$\lambda^{*}$ on $L$ such that $u\left(\lambda^{*}\right)>2 m n \pi /(b-a)$. Using the monotone increasing nature of $\beta_{j}(b, \lambda), j=1,2, \ldots, n$, in $\lambda, \lambda<\lambda^{*}$ and there exist $k+1$ values of $\lambda, \lambda<\lambda^{0}<\lambda^{1}<\cdots$ $<\lambda^{k}<\lambda^{*}$, such that

$$
\sum_{j=1}^{n} \beta_{j}\left(b, \lambda^{p}\right)=2(h+n+p n) \pi, \quad p=0,1, \ldots, k-1
$$

and

$$
2[h+n+p n] \pi<\sum_{j=1}^{n} \beta_{j}(b, \lambda) \leqq 2[h+n+(p+1) n] \pi
$$

for $\lambda^{p}<\lambda \leqq \lambda^{p+1}$. Since $\sum_{j=1}^{n} \beta,(b, \lambda)$ increases by $2 n \pi$ as $\lambda$ increases from $\lambda^{p}$ to $\lambda^{p+1}$, at least one $\beta_{j}(b, \lambda)$ increases by $2 \pi$ and, consequently, there exists at least one value of $\lambda, \lambda^{p}<\lambda \leqq \lambda^{p+1}$ such that $\beta_{j}(b, \lambda) \equiv 0(\bmod 2 \pi)$. Let $J_{p}$ be the set of all $\lambda$ on $\lambda^{p}<\lambda \leqq \lambda^{p+1}$ such that $\beta_{j}(b, \lambda) \equiv 0(\bmod 2 \pi)$ for at least one $j$.

Corollary 1. Let the hypotheses of the theorem be satisfied. Each set $J_{p}$, $0 \leqq p \leqq k-1$, contains at most $2 n-1$ distinct eigenvalues. Moreover, if $\lambda_{p} \in J_{p}$, $p \geqq 1$, then $\operatorname{det} Y\left(x, \lambda_{p}\right)$ has at least $p-1$ and at most $n+[h+2 n+(p+1) n]-1$ zeros on $X$, multiple zeros counted according to their multiplicity.

Proof. Since $\sum_{j=1}^{n} \beta_{j}(b, \lambda)$ is increasing in $\lambda$ and since $\sum_{j=1}^{n} \beta_{j}(b, \lambda)$ increases by $2 n \pi$ on each interval $\lambda^{p}<\lambda \leqq \lambda^{p+1}$ there are at most $2 n-1$ distinct values of $\lambda$ such that $\beta_{j}(b, \lambda) \equiv 0(\bmod 2 \pi)$. The estimates for the number of zeros of $\operatorname{det} Y\left(x, \lambda_{p}\right), \lambda_{p} \in J_{p}$, on $X$ follows from the inequalities

$$
2[h+n+p n] \pi<\sum_{j=1}^{n}\left[w_{j}\left(b, \lambda_{p}\right)-w_{j}\left(a, \lambda_{p}\right)\right]<2[h+n+(p+1) n] \pi+2 n \pi
$$

and the results in $[6, \S 3]$.

COROLlaRY 2. Under the hypotheses of the theorem, if the integer $m$ may be chosen arbitrarily large, then there exist infinitely many nonempty sets, $J_{0}, J_{1}, \ldots$, of eigenvalues for the system (1.1), (1.2).

The theorem and the corollaries may also be restated as follows.

COROLLARY 3. Let the hypotheses of the theorem hold. Then there is an increasing (finite) sequence $\lambda_{0}, \lambda_{1}, \ldots$ of eigenvalues and a nondecreasing (finite) sequence of nonnegative integers $h_{0}, h_{1}, \ldots$, such that det $Y\left(x, \lambda_{p}\right)$ has exactly $h_{p}$ zeros on $X$ multiple zeros being counted according to their multiplicity. Moreover, if the integer $m$ may be chosen arbitrarily large, then the sequence of eigenvalues $\lambda_{0}, \lambda_{1}, \ldots$ is infinite and the corresponding sequence of nonnegative integers $h_{0}, h_{1}, \ldots$ has the property that $h_{p} \rightarrow \infty$ as $p \rightarrow \infty$.

To pursue the analogy with Whyburn's work [7], [15], we introduce a hypothesis which will insure a monotone behavior in $x$ in the solution $\{Y(x, \lambda), Z(x, \lambda)\}$ of (1.1), (2.1) and in the pair $\{U(x, \lambda), V(x, \lambda)\}$ defined in (3.6). 
H.13. $G(x, \lambda)>0$ on $X L$.

Using the technique employed by Atkinson [1], it is readily verified that the positive definiteness of $K$ and $G$ on $X L$ implies that, for each $\lambda$ on $L$, each of the characteristic root functions $\phi_{j}(x, \lambda)$ and $f_{j}(x, \lambda), j=1,2, \ldots, n$ of $\theta(Y, Z) \equiv E(x, \lambda)$ and $F(x, \lambda)$, respectively, move around the unit circle in the positive direction as $x$ increases on $X$. Consequently, each of the argument functions $w_{j}(x, \lambda)$ and $\beta_{j}(x, \lambda)$ is increasing on $X$ for each fixed $\lambda$ on $L$.

THEOREM 4.4. Let H.1-H.13 hold. Let $\{Y(x, \lambda), Z(x, \lambda)\}$ be the solution of (1.1), (2.1) and let $h$ be the smallest integer such that g.l.b. of $v(\lambda)$ on $L$ is less than $2 h \pi /(b-a)$. If $m$ is an integer such that l.u.b. of $u(\lambda)$ on $L$ is greater than $2 m n \pi /(b-a)$ and such that $m>h$ with $k$ the largest nonnegative integer having the property $m-h>k n$, then there exist at least $k$ nonempty sets of eigenvalues $D_{0}, D_{1}, \ldots, D_{k-1}$ for the system (1.1), (1.2). Moreover, for each $p, p=0,1, \ldots, k-1, D_{p}$ contains at most $2 n-1$ distinct eigenvalues and if $\lambda_{p} \in D_{p}$, then each of $\operatorname{det} Y\left(x, \lambda_{p}\right)$ and $\operatorname{det} Z\left(x, \lambda_{p}\right)$ has at least $p$ and at most $n+[h+n+(p+1) n]-1$ zeros on $X$, multiple zeros being counted according to their multiplicity.

Proof. Assuming that the hypotheses of the theorem hold and that $h$ and $m$ are the integers so described, select $\lambda$ and $\lambda^{*}$, as in Theorems 4.1 and 4.3.

Since the functions $\beta_{j}(x, \lambda), j=1,2, \ldots, n$, are increasing in both $x$ and $\lambda$, we can conclude that $\sum_{j=1}^{n}\left[\beta_{j}(b, \lambda)-\beta_{j}(a, \lambda)\right]>0$ on $L$, and, in fact, $\sum_{j=1}^{n} \beta_{j}(b, \lambda)>0$ on $L$. In view of the monotone increasing behavior of $\sum_{j=1}^{n} \beta_{j}(b, \lambda)$ in $\lambda$ and the fact that $\sum_{j=1}^{n} \beta_{j}(b, \lambda)<2 h \pi$ and $\sum_{j=1}^{n} \beta_{j}\left(b, \lambda^{*}\right)>2 m n \pi$ with $m-h \geqq k n$, there exist $k+1$ values of $\lambda, \lambda^{0}<\lambda^{1}<\cdots<\lambda^{k}$, such that

$$
\sum_{j=1}^{n} \beta_{j}\left(b, \lambda^{p}\right)=2[h+p n] \pi, \quad p=0, \ldots, k-1 .
$$

On each interval $\lambda^{p}<\lambda \leqq \lambda^{p+1}, \sum_{j=1}^{n} \beta_{j}(b, \lambda)$ increases by $2 n \pi$ and, consequently, there is at least one $\lambda_{p}$ such that $\beta_{j}\left(b, \lambda_{p}\right) \equiv 0(\bmod 2 \pi)$ and at most $2 n-1$ such values of $\lambda$. As noted previously, each such $\lambda$ is an eigenvalue for the system. Denote by $D_{p}$ the totality of such $\lambda$ 's on $\lambda^{p}<\lambda \leqq \lambda^{p+1}$.

The estimates for the number of zeros of each of $\operatorname{det} Y\left(x, \lambda_{p}\right)$ and $\operatorname{det} Z\left(x, \lambda_{p}\right)$, $\lambda_{p} \in D_{p}$, on $X$ follows, as in Corollary 1 of Theorem 4.3, from the inequalities

$$
2 p n \pi<\sum_{j=1}^{n}\left[w_{j}\left(b, \lambda_{p}\right)-w_{j}\left(a, \lambda_{p}\right)\right]<2[h+(p+1) n] \pi+2 n \pi
$$

and the results in $[6, \S 3]$.

Again, there is the immediate corollary:

COROLLARY 1. Under the hypotheses of the theorem, if the integer $m$ may be chosen arbitrarily large, then there exist infinitely many nonempty sets of eigenvalues, $D_{0}, D_{1}, \ldots$, for the system (1.1), (1.2). 
We conclude by remarking that the matrix Sturm-Liouville two point boundary problem consisting of the matrix equation

$$
\left(P(x) Y^{\prime}\right)^{\prime}+(\lambda F(x)+Q(x) Y)=0
$$

on $X: a \leqq x \leqq b, L:-\infty<\lambda<\infty$, where $P(x), F(x)$ and $Q(x)$ are $n \times n$ continuous symmetric matrices on $X$ with $P>0$ and $F>0$, together with the boundary conditions

$$
\begin{aligned}
\bar{A} Y(a, \lambda)-\bar{B}\left(P(a) Y^{\prime}(a, \lambda)\right) & =0, \\
\operatorname{det}\left[\bar{\Gamma} Y(b, \lambda)-\bar{\Delta}\left(P(b) Y^{\prime}(b, \lambda)\right)\right] & =0,
\end{aligned}
$$

where $\bar{A}, \bar{B}, \bar{\Gamma}$ and $\bar{\Delta}$ are $n \times n$ constant matrices satisfying H.3-H.6, is a special case of the system (1.1), (1.2). It has been considered in [1, Chapter 10, §5]. Using the methods and results of the preceding work, we can obtain the following

THEOREM 4.5. There are an infinite number of eigenvalues $\lambda_{0}, \lambda_{1}, \ldots$ for the problem (4.3), (4.4) forming an increasing sequence with $\lambda_{m} \rightarrow \infty$ as $m \rightarrow \infty$. Moreover, there is a nondecreasing sequence $h_{0}, h_{1}, \ldots$ of nonnegative integers with $h_{m} \rightarrow \infty$ as $m \rightarrow \infty$ such that if $\{Y(x, \lambda), Z(x, \lambda)\}$ is any nontrivial, conjoined solution of (4.3), (4.4) then $\operatorname{det} Y\left(x, \lambda_{m}\right)$ has exactly $h_{m}$ zeros on $X$, multiple zeros counted according to their multiplicity.

We omit the proof of this theorem since it is also readily obtained through the use of the calculus of variations. We refer to M. Morse [10].

\section{REFERENCES}

1. F. V. Atkinson, Discrete and continuous boundary problems, Academic Press, New York, 1964. MR 31 \#416.

2. J. H. Barrett, A Prüfer transformation for matrix differential equations, Proc. Amer. Math. Soc. 8 (1957), 510-518. MR 19, 415.

3. E. A. Coddington and N. Levinson, Theory of ordinary differential equations, McGrawHill, New York, 1955. MR 16, 1022.

4. G. J. Etgen, Oscillatory properties of certain nonlinear matrix differential systems of second order, Trans. Amer. Math. Soc. 122 (1966), 289-310. MR 32 \#7834.

5. - A note on trigonometric matrices, Proc. Amer. Math. Soc. 17 (1966), 1226-1232. MR 35 \#4504.

6. - On the oscillation of solutions of second order, self-adjoint matrix differential equations, J. Differential Equations 6 (1969).

7. A. K. Hinds and W. M. Whyburn, A non-self-adjoint differential system of the second order, J. Elisha Mitchell Sci. Soc. 68 (1952), 32-43. MR 14, 556.

8. E. L. Ince, Ordinary differential equations, Longmans, Green \& Co., 1926; reprint, Dover, New York, 1944. MR 6, 65.

9. V. A. Jakubovič, Oscillatory properties of solutions of canonical equations, Mat. Sb. 56 (98) (1962), 3-42; English transl., Amer. Math. Soc. Transl. (2) 42 (1964), 247-288. MR 25 \#2303.

10. M. Morse, The calculus of variations in the large, Amer. Math. Soc. Colloq. Publ., vol. 17, Amer. Math. Soc., Providence, R. I., 1934.

11. W. T. Reid, Boundary value problems of the calculus of variations, Bull. Amer. Math Soc. 43 (1937), 633-666. 
12. W. T. Reid, An integro-differential boundary value problem, Amer. J. Math. 60 (1938), 257-292.

13. — A Prüfer transformation for differential systems, Pacific J. Math. 8 (1958), 575584. MR $20 \# 5913$.

14. W. M. Whyburn, Existence and oscillation theorems for non-linear differential equations of second order, Trans. Amer. Math. Soc. 30 (1928), 848-854.

15. - A nonlinear boundary value problem for second order differential systems, Pacific J. Math. 5 (1955), 147-160. MR 16, 1027.

UNIVERSITY OF HOUSTON,

Houston, TeXas 77004 